\title{
Elisabeth Selbert: „Männer und Frauen sind gleichberechtigt"
}

60 Jahre djb - der runde Geburtstag lenkt den Blick auf das Gründungsjahr 1948 und die Nachkriegsjahre, in deren Kontext der Deutsche Juristinnenbund sich als Nachfolgeorganisation des 1914 gegründeten „Deutschen Juristinnen-Vereins“ neu formierte. $\mathrm{Zu}$ den ersten neuen Mitgliedern gehörte neben den späteren Bundesverfassungsrichterinnen Erna Scheffler und Wiltraud Rupp-von Brünneck, der späteren Bundestagsabgeordneten Elisabeth Lüders und der später ersten Bundesministerin in der Geschichte der Bundesrepublik Deutschland, Elisabeth Schwarzhaupt, auch die Rechtsanwältin und Notarin Elisabeth Selbert, seinerzeit Mitglied des Hessischen Landtages, des zentralen Parteivorstandes der SPD und neben weiteren Ämtern auch Stadtverordnete in Kassel. Ihrem couragierten Engagement und gezielten Appell an die Solidarität der Frauen unter Nutzung der ihr zur Verfügung stehenden Netzwerke ist es zu verdanken, dass der Grundsatz „Männer und Frauen sind gleichberechtigt“ als Artikel 3 Absatz 2 im Grundgesetz an prominenter Stelle als unveräußerliches und nicht disponibles Grund- und Menschenrecht verankert ist. Ohne diesen gleichsam „Grundstein“ ist die Entwicklung der Verrechtlichung der Stellung der Frau in allen Lebensbereichen seit dem 2. Weltkrieg nicht denkbar; das weitere große Jubiläum in diesem Jahr - 50 Jahre Gleichberechtigungsgesetz - hätte es sicher so nicht gegeben.

Elisabeth Selbert ist es zu verdanken, dass der Satz „Männer und Frauen sind gleichberechtigt“ heute für uns Nachgeborene so selbstverständlich und mit Verfassungsrang daherkommt. Er ist den Frauen nicht geschenkt worden, nicht von den Männern, und - jedenfalls zunächst - auch nicht von den Frauen. Elisabeth Selbert hat ihn erkämpft und gegen immense Widerstände durchgesetzt, mit öffentlichem Druck erzwungen.

Dabei war ihr die Frauenrechtlerin zunächst nicht in die Wiege gelegt. Als zweite von vier Töchtern wurde Martha Elisabeth Rohde am 22. September 1896 in Kassel geboren. Sie wuchs in einer kleinbürgerlichen, streng protestantisch geprägten Familie auf. Der Vater war Aufseher in einer Kasseler Jugendstrafanstalt, die Mutter versorgte den Haushalt und die vier Kinder. Trotz dieser traditionellen Rollenverteilung soll die Familie nicht vom Vater dominiert worden, die fünf Frauen des Hauses starke Persönlichkeiten gewesen sein; die Selbstverständlichkeit, mit der Elisabeth als junge
Frau ihre berufliche und politische Bildung in die Hand nehmen wird, spricht ebenso wie die spätere Unterstützung durch ihre Eltern dafür, dass ihr Selbstverständnis als gleichberechtigte Frau im Elternhaus angelegt und gewachsen ist.

Gleichwohl unterliegt sie der für die damalige Zeit typischen schulischen Erziehung. Die Ausbildung der Mädchen und jungen Frauen ist am patriarchalischen Familienbild und Rollenverständnis ausgerichtet. In Elisabeths Geburtsjahr 1896 wird das Bürgerliche Gesetzbuch verabschiedet, das am 1. Januar 1900 in Kraft tritt und bis in die 70er Jahre des anbrechenden 20. Jahrhunderts dem Ehemann das alleinige Entscheidungsrecht in Ehe und Familie einräumen wird. Zwar ist Elisabeth eine gute Schülerin, die nach der Volksschule auf die Realschule wechselt und aufgrund ihrer guten schulischen Leistungen sogar von der Schulgeldzahlung befreit wird. Ein Zeugnis und die Mittlere Reife erhält sie aber nach Beendigung der Schule nicht: Zeugnisse werden nur den Jungen erteilt, was die damals Sechzehnjährige als erste einschneidende, geschlechtsspezifische Diskriminierung erfahren muss. Das Schulgeld für die danach mögliche Fortsetzung der Ausbildung an einer höheren Mädchenschule kann die Familie nicht aufbringen, und so besucht sie ab 1912 die Kasseler Gewerbe- und Handelsschule des Frauenbildungsvereins. Sie möchte gerne Lehrerin werden, einer der wenigen für Frauen offenen Berufe, aber auch dieses Ziel scheitert an den beengten finanziellen Verhältnissen der Familie. So erlernt Elisabeth Fremdsprachen und wird mit 17 Jahren Auslandskorrespondentin bei einer Kasseler Import-Exportfirma. Nur ein Jahr später verliert sie diese Arbeit unmittelbar nach Ausbruch des 1. Weltkrieges.

Der durch den Krieg bedingte Bedarf an Männern lässt Arbeitsstellen auch in zuvor männlich dominierten Berufen frei werden, und ab 1916 arbeitet Elisabeth als Postbeamtenanwärterin im Telegrafendienst der Reichspost. Hier lernt sie im Alter von 22 Jahren, während der sogenannten Novemberrevolution 1918, ihren späteren Ehemann, Adam Selbert, kennen. Er ist gelernter Buchdrucker und Vorsitzender des Arbeiter- und Soldatenrates in Niederzwehren bei Kassel. Adam Selbert fördert ihr politisches Interesse und nimmt sie zu Veranstaltungen mit. Ende 1918 wird Elisabeth Mitglied der SPD und umgehend auch des Bezirksvorstandes in Kassel.

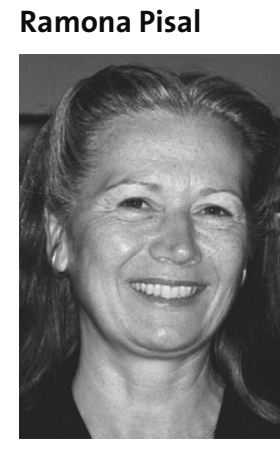

Vizepräsidentin des djb, Vors. Richterin am OLG, Brandenburg a.d.H. 
Auch Philipp Scheidemann, der spätere Reichskanzler, seinerzeit Oberbürgermeister in Kassel, ermutigt Elisabeth, selbst politisch aktiv zu werden. Mit der Weimarer Reichsverfassung haben Frauen seit 1919 das aktive und passive Wahlrecht. Nach erfolgreicher Kandidatur für das Gemeindeparlament lässt sie sich in den Finanz- und Steuerausschuss wählen, um der damals wie heute üblichen Zuschreibung eines „weich“ genannten Ressorts, der Fürsorge, zu entgehen. Elisabeth publiziert Artikel und spricht auf zahlreichen Veranstaltungen über die Pflicht der Frauen, sich politisch zu informieren und zu engagieren. Trotz der Arbeit im Finanzausschuss bleibt ihr Thema die Gleichberechtigung. Artikel 109 der Weimarer Verfassung bestimmt zwar, dass Männer und Frauen grundsätzlich die gleichen staatsbürgerlichen Rechte und Pflichten haben, ändert aber damit nichts an der durch das Bürgerliche Gesetzbuch manifestierten männlichen Dominanz: Danach hat der Ehemann das Letztentscheidungsrecht in allen Eheangelegenheiten und Vorrechte in Erziehungsfragen die gemeinsamen Kinder betreffend. Er allein ist berechtigt, über das Vermögen und die Einkünfte der Ehefrau zu verfügen, er kann ein Dienstverhältnis der Ehefrau eigenmächtig kündigen, ohne seine Einwilligung darf sie kein Dienstverhältnis eingehen. Die Aufgabenverteilung in der Ehe ist gesetzlich vorgeschrieben. Die Frau verliert mit der Heirat ihren Namen.

Im Oktober 1920, kurz nach ihrer Heirat mit Adam Selbert, nimmt sie als Delegierte an der ersten Reichsfrauenkonferenz in Kassel teil und kritisiert

„... dass wir zwar heute die Gleichberechtigung für unsere Frauen haben, dass aber diese Gleichberechtigung immer noch eine rein papierne ist. Wir müssen nun dabin wirken, dass die Gleichberechtigung in der Praxis bis zur letzten Konsequenz durchgeführt wird..."

Elisabeth bekommt zwei Kinder, arbeitet trotzdem weiter als Beamtin im Telegrafenamt und betätigt sich auch weiterhin politisch. Zunehmend stellt sie fest, dass ihr für eine fundierte politische Arbeit die theoretischen Grundlagen fehlen, insbesondere im Staats- und Verfassungsrecht. Sie hofft, dass

„juristische Ausbildung helfen würde, politisch effizienter wirken zu können."

Adam Selbert bestärkt seine Frau in ihren Fortbildungsbestrebungen, die auch bei den Eltern, Schwiegereltern und einer Schwester auf Akzeptanz treffen und Unterstützung erfahren. Im Selbststudium bereitet sich Elisabeth auf das Abitur vor, die Reifeprüfung besteht sie 1926 an der Luisenschule in Kassel als Externe. Sie ist jetzt 30 Jahre alt, ihre beiden Söhne, vier und fünf Jahre alt, werden von ihrem Mann und den Eltern versorgt.

Sie studiert zunächst in Marburg als einzige Frau Rechtsund Staatswissenschaften, nach dem Wechsel an die Universität Göttingen - weil sich in Marburg kein Doktorvater findet - ist sie unter den ca. 300 Studenten immerhin eine von fünf Frauen. Sie selbst stört der Männerüberhang angeblich wenig, aber ihre Professoren scheinen mitunter überfordert: Elisabeth Selbert und ihre Kommilitoninnen werden zum Beispiel gebeten, den Hörsaal zu verlassen, wenn der Professor über Sexu- aldelikte spricht. Nach nur sechs Semestern schließt sie das Studium mit Auszeichnung ab und promoviert schon ein Jahr später mit dem Thema „Ehezerrüttung als Scheidungsgrund“. Sie kritisiert das Schuldprinzip, das die Frauen bei der Scheidung quasi rechtlos stellt, tritt ein für eine „Entgiftung“ des Scheidungsprozesses und ist mit ihrer Forderung nach dem Zerrüttungsprinzip im Jahre 1930 ihrer Zeit weit voraus. Ihre Vorschläge sollten in Deutschland erst mit der Eherechtsreform 1977, als sie 80 Jahre alt war, aufgegriffen und umgesetzt werden.

1933 kandidiert Elisabeth Selbert auf der hessischen Landesliste der SPD erfolglos für den Reichstag. Nach der „Machtübernahme“ der Nationalsozialisten verliert Adam Selbert seine Arbeit und wird in „Schutzhaft“ genommen. Nach seiner Entlassung darf er nicht wieder arbeiten, die Gestapo hat die Familie im Visier. Als Elisabeth 1934 das zweite Staatsexamen mit Prädikat abgelegt hat, stellt sie kurz darauf, auf Drängen ihres Mannes, den Antrag auf Zulassung zur Anwaltschaft, denn es ist absehbar, dass sie mit ihrem Einkommen den Familienunterhalt sicherstellen muss. Und der Antrag eilt, weil die neuen Machthaber keine Frauen in der Rechtspflege dulden. Otto Palandt, überzeugter Nationalsozialist, zuvor Präsident des Landgerichts Kassel, wird Präsident des Reichsjustizprüfungsamtes und damit neben der Juristenausbildung auch zuständig für die Zulassung zu den juristischen Berufen. Jede außer Haus tätige Frau wird als Volksfeindin angesehen, erklärtes Ziel ist es, den Frauenanteil an den Studierenden auf unter 10 Prozent zu drücken. Richterin oder Staatsanwältin kann schon nach der „Machtergreifung“ keine Frau mehr werden, und mit dem 15. Januar 1935 wird auch die Zulassung zur Rechtsanwältin verboten, weil das einen „Einbruch in den altgeheiligten Grundsatz der Männlichkeit des Staates“ bedeutet hätte.

Auch Elisabeth Selberts Antrag steht zunächst unter keinem guten Stern. Der Wille des nationalsozialistischen Präsidenten, das Votum der Rechtsanwaltskammer, die Entscheidung des Gauleiters und des NS-Juristenbundes stehen der Bewilligung entgegen. Gleichwohl wird sie am 15. Dezember 1934 als letzte Frau zur Anwaltschaft zugelassen. Es sollen zwei ältere Senatspräsidenten gewesen sein, die sich für Selbert einsetzten und in Vertretung für den im Urlaub befindlichen Oberlandesgerichtspräsidenten ihre Zulassung unterzeichneten. Die Kanzlei, die Elisabeth Selbert nun eröffnet, übernimmt sie von den jüdischen Rechtsanwälten Karl Elias und Leon Rossmann, die von den Nationalsozialisten zur Aufgabe ihrer Sozietät und dann auch zur Emigration gezwungen werden. Der für sie finanziell günstige Karrierestart wird ihr später vorgeworfen werden. Sie bearbeitet vor allem Wirtschaftsstrafsachen, auch, nachdem die Büroräume 1943 durch einen Luftangriff zerstört worden sind. Sie befasst sich aber auch mit Fällen der Verweigerung der Arbeitsdienstpflicht sowie mit den vermögensrechtlichen Seiten der Judenverfolgung und als Scheidungsanwältin mit der nationalsozialistischen Rassenpolitik. Wiederholt muss sie sich wegen angeblich staatsfeindlicher Äußerungen verantworten und lebt in großer Angst 
um ihre eigene Existenz und die ihrer Familie. Ihr Mann bleibt bis 1945 erwerbslos, sie ernährt die Familie.

Nach dem Ende des 2. Weltkrieges wird Elisabeth Selbert erneut politisch aktiv. Sie arbeitet im Ausschuss zur Neuordnung der Justizverwaltung und als Stadtverordnete in Kassel, gehört dem Bezirksvorstand ihrer Partei an, wird zum Mitglied der Verfassungsberatenden Landesversammlung in Hessen berufen. Daneben führt sie ihre Kanzlei, inzwischen auch als Notariat, weiter. Die schwierigste und folgenreichste Aufgabe liegt aber noch vor ihr: Die Mitarbeit am Entwurf eines Grundgesetzes für die mit seiner Inkraftsetzung zu gründende Bundesrepublik Deutschland.

Die Grundzüge der zu erarbeitenden Verfassung, die aus Gründen der deutschen Teilung nur eine vorläufige sein und daher auch nicht als solche, sondern als Grundgesetz bezeichnet werden sollte, hatte bereits der Verfassungskonvent auf Herrenchiemsee in nur zwei Wochen, zwischen dem 10. und dem 25. August 1948, entworfen. Ihm hatte keine Frau angehört; die elf Länder im Westen hatten mehrheitlich Minister, Staatssekretäre und Rechtswissenschaftler entsandt. Auch unter den Mitarbeitern befand sich keine Frau. Zwar hatten die Frauen in den Kriegsjahren und danach auf sich gestellt schwerste Arbeit erfolgreich geleistet und bislang den Männern vorbehaltene Bereiche über fast ein Jahrzehnt besetzt, aber die Mitwirkung an der Gestaltung und rechtlichen Ausstattung des künftigen Staates sollte ihnen vorenthalten werden. Die Männer waren zurück, die Frauen hatten ihren Dienst getan. Dabei war der Frauenanteil aufgrund der Kriegsverluste erheblich höher, es gab sieben Millionen mehr Frauen als Männer, auf 100 Wähler kamen 170 Wählerinnen.

Elisabeth Selbert war politisch unbelastet und mit der amerikanischen Besatzungsmacht durch ihre politische Arbeit und ihre Sprachkenntnisse bald auch persönlich bekannt. Als auf Anweisung der westlichen Siegermächte, insbesondere der Amerikaner, die Ministerpräsidenten der elf Länder der Westzone am 1. September 1948 den Parlamentarischen Rat einsetzten, wurde Elisabeth Selbert in dieses hohe Gremium berufen, das die Aufgabe hatte, das neue Grundgesetz für die Bundesrepublik Deutschland auszuarbeiten. Sie war eine von nur vier Frauen - mit Frieda Nadig (SPD), Helene Weber (CDU) und Helene Wessel (Zentrum) - unter den insgesamt 65 stimmberechtigten Mitgliedern. Insbesondere der amerikanischen Besatzungsmacht gefiel diese Unterrepräsentanz von Frauen nicht.

Der Kampf um den Gleichberechtigungsgrundsatz kam für Elisabeth Selbert in diesem Ausmaß überraschend. Sie war in verschiedenen Ausschüssen tätig, wie auch die anderen drei Frauen, sie verstand sich nicht als Frauenrechtlerin und hatte angenommen, die Zeit sei über die überkommenen Rollenbilder längst hinweg. Als sie aber merkte, dass mit dem ursprünglichen Formulierungsentwurf für Artikel 3, wortgleich mit Artikel 109 der Weimarer Verfassung: „Männer und Frauen haben die gleichen staatsbürgerlichen Rechte und Pflichten“ die Gleichberechtigung der Frau im Verhältnis zum Mann keinen Eingang in das Grundgesetz finden sollte, schal- tete sie sich ein. Auch die alternative Formulierung „Der Gesetzgeber muß Gleiches gleich, Verschiedenes nach seiner Eigenart behandeln “ wollte sie nicht akzeptieren, denn damit wäre die Diskriminierung der Frauen aufgrund vermeintlich natürlich gegebener Unterschiede auch noch legalisiert worden. Elisabeth Selbert wollte die Gleichberechtigung „als imperativen Auftrag an den Gesetzgeber“ verstanden wissen, die Gleichberechtigung sollte nach ihrem Vorschlag mit dem Wortlaut „Männer und Frauen sind gleichberechtigt“ als Verfassungsgrundsatz aufgenommen werden.

In Konsequenz dieser Formulierung zeichnete sich eine weitgehende Überarbeitung des BGB ab, da es in vielen insbesondere familienrechtlichen Bestimmungen seit 1896 diesem Grundsatz widersprach, man befürchtete ein Rechtschaos. Darum und weil damit gleichzeitig die patriarchale Gesellschaftsordnung an sich gefährdet war, stieß der Vorschlag auf fast einhellige Ablehnung, auch bei den drei weiblichen Delegierten, sogar bei der Kollegin aus der eigenen Partei. In der ersten Lesung wird die Formulierung abgelehnt.

Elisabeth Selbert gibt nicht auf. Um dem Argument des Rechtschaos zu begegnen, erarbeitet sie zusammen mit Wiltraut Rupp-von Brünneck die Übergangsvorschrift Artikel 117 Grundgesetz, wonach dem Gesetzgeber eine Frist bis 31. März 1953 zur Anpassung der dem Gleichheitsprinzip entgegenstehenden Gesetze eingeräumt wird. Sie argumentiert innerhalb ihrer Partei, droht mit der Mobilisierung der Öffentlichkeit, setzt das zahlenmäßige Potential der künftigen Wählerinnen ein. Die konservativen Einwände - die natürlichen, i. e. biologischen Unterschiede zwischen den Geschlechtern seien einer Gleichberechtigung in der Ehe konträr - kann sie argumentativ nicht überwinden. Nach erneuter Ablehnung in zweiter Lesung macht sie ihre Ankündigung wahr und wendet sich an die Öffentlichkeit.

Durch ihre Parteiarbeit verfügt sie über vielfältige Verbindungen zu Frauenverbänden und Gewerkschaftsfrauen. An sie appelliert sie, sich mit ihr für die Aufnahme der Gleichberechtigung in das Grundgesetz einzusetzen und klärt sie über die Tragweite der Regelung auf. Eine breite Öffentlichkeit, die so auch erstmals auf die Arbeit des Parlamentarischen Rates überhaupt aufmerksam wird, reagiert mit einer ungeheuren Resonanz. Waschkörbeweise gehen Protestschreiben der weiblichen Landtagsabgeordneten, Gewerkschafterinnen, Frauenverbände, zigtausender Metall- und anderer Arbeiterinnen bei den Parlamentariern aller Parteien ein. Die Drohkulisse einer Ablehnung der gesamten Verfassung über die Gleichberechtigungsfrage baut sich auf. Ihre Parteikollegin im Parlamentarischen Rat unterstützt Selberts Formulierung danach aus eigener Überzeugung:

„In aller Öffentlichkeit gilt es inzwischen, für die Gleichberechtigung der Frau einzutreten und sie darauf hinzuweisen, welches alte Unrecht verewigt werden soll. Das Übergewicht von sieben Millionen Frauen bedeutet eine gewaltige Macht, die für das Recht voll eingesetzt werden muß."

Einen konservativen Kompromissvorschlag „Männer und Frauen haben die gleichen Rechte und Pflichten“ auf allen 
Rechtsgebieten lehnt Selbert ab, weil sie darin den Mann als Maßstab der Rechte und Pflichten und die Frau als hiervon abgeleitet erkennt. Das zähe Ringen geht weiter, sie gibt nicht auf, muss aushalten, dass ihr von Theodor Heuss spöttisch die Kompetenz abgesprochen wird, der die Protestwelle als „Quasi-Stürmlein " kleinreden will. Aber am Ende setzt sie sich durch: Am 18. Januar 1949 wird Artikel 3 Absatz 2 GG in dem von ihr vorgeschlagenen Wortlaut ohne jede Gegenstimme angenommen. Einen Tag später wendet sie sich in einer eindrucksvollen Rundfunkansprache an die Bevölkerung:

„... dieser Tag war ein geschichtlicher Tag, eine Wende auf dem Wege der deutschen Frauen der Westzonen. Lächeln Sie nicht! Es ist nicht falsches Pathos einer Frauenrechtlerin, das mich so sprechen läßt. Ich bin Jurist und unpathetisch und Frau und Mutter und zu solchen frauenrechtlerischen Dingen gar nicht geeignet..."

Auch in der Folgezeit mag sie sich nicht auf Frauenfragen festlegen lassen. Im Unterschied zu den anderen drei „Müttern “ des Grundgesetzes scheitert ihr Einzug in den Ersten Deutschen Bundestag, wenn auch knapp. Sie erhält 1956 das Große Bundesverdienstkreuz, bleibt noch bis 1958 in der Landespolitik, konzentriert sich danach aber auf ihre Kanzlei und gerät in der Öffentlichkeit in Vergessenheit. Adam Selbert stirbt im Jahr 1968. Bis über das achtzigste Lebensjahr hinaus geht sie mit großem Einsatz ihrer anwaltlichen Arbeit nach. Am 9. Juni 1986 verstirbt sie in ihrer Heimatstadt Kassel wenige Monate vor ihrem 90. Geburtstag.

„Ich hatte einen Zipfel der Macht in meiner Hand gehabt und den habe ich ausgenutzt, in aller Tiefe, in aller Weite, die mir rhetorisch zur Verfügung stand. Es war die Sternstunde meines Lebens, als die Gleichberechtigung der Frau damit zur Annabme kam."

\section{Literatur}

Böttger, Barbara: Das Recht auf Gleichheit und Differenz. Elisabeth Selbert und der Kampf der Frauen um Art. 3 II Grundgesetz, Münster, 1990; Dertinger, Antje: Elisabeth Selbert. Eine Kurzbiographie, Wiesbaden 1986; dies.: Männer und Frauen sind gleichberechtigt. Die Juristin Elisabeth Selbert erstritt Art. 3 II, in: dies.: Frauen der ersten Stunde. Aus den Gründungsjahren der Bundesrepublik, Bonn 1989; S. 179-190; Drummer, Heike/Zwilling, Jutta: Elisabeth Selbert. Eine Biographie, in: Hessische Landesregierung (Hrsg.): „Ein Glücksfall für die Demokratie“. Elisabeth Selbert (1896-1986). Die große Anwältin der Gleichberechtigung, Frankfurt/Main 1999; Mundzeck, Heike: Elisabeth Selbert, in: Deutscher Juristinnenbund (Hrsg.): Juristinnen in Deutschland. Die Zeit von 1900 bis 1998, Baden-Baden 1998, S. 18-194; Nachlass Elisabeth Selbert, Stiftung Archiv der deutschen Frauenbewegung, Kassel; Notz, Giesela: Frauen in der Mannschaft. Sozialdemokratinnen im Parlamentarischen Rat und im Deutschen Bundestag 1948/49 bis 1957. Bonn 2003, S. 16 ff.; Röwekamp, Marion: Juristinnen - Lexikon zu Leben und Werk, Baden Baden 2005, S. 395-399.

\section{„Erzählte Geschichte“ in Frauenverbänden}

\section{Ein Projekt der Stiftung Archiv der deutschen Frauenbewegung}

Der Deutsche Juristinnenbund wurde 1948 gegründet, er geht ursprünglich auf den Deutschen Juristinnen-Verein von 1914 zurück, ist also inzwischen 60 Jahre alt bzw. ideell sogar schon fast 100 Jahre - eine lange Geschichte. Je länger die Geschichte eines Verbandes währt, um so mehr stellt sich die Frage nach ihrer Überlieferung. Doch wie entsteht Überlieferung? Woraus bildet sich das, was schließlich als „die Verbandsgeschichte“ in die Annalen eingeht?

Zum einen sicherlich aus den Akten, die im Laufe der Jahre und Jahrzehnte entstehen (und hoffentlich aufbewahrt werden!). Darin finden sich die Korrespondenzen, die Protokolle der Mitgliederversammlungen und der Sitzungen von Vorständen, Kommissionen und Arbeitsgruppen, Presseerklärungen, Petitionen und andere Verlautbarungen und schließlich auch Unterlagen über die finanzielle Seite wie Bilanzen, Kassen- und Kontoaufstellungen und vieles mehr. Hier finden wir also in erster Linie die schriftliche Überlieferung und - wenn es gut geht - auch eine bildliche in Form von Fotos oder gar Filmen, die zu besonderen Anlässen gemacht und aufbewahrt wurden. All dies wird gelegentlich ergänzt durch Sammlungen einzelner Protagonistinnen des Verbandes, die diese möglicherweise dem Verbandsarchiv übergeben oder hinterlassen.

Ein weiterer, oft vernachlässigter Bereich der historischen Überlieferung sind die persönlichen Erinnerungen der Mitglieder, die es zum Beispiel in Form von Tagebuchaufzeichnungen, schriftlicher Niederlegung der Erinnerungen und in Form von Interviews gibt. Sie ergänzen die vorgenannten, eher sachlichen Belege und beleben sie auch in gewisser Weise. „Erzählte Geschichte“ ist immer eine höchst subjektive Sichtweise einzelner Menschen, die aber dennoch, richtig abgewägt und eingeord-

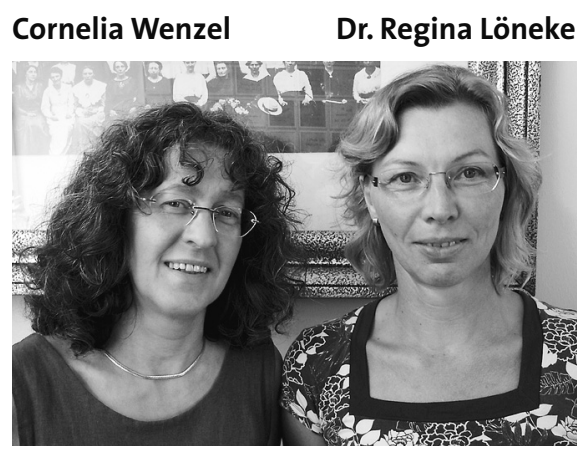

Archiv der deutschen Frauenbewegung, Kassel 